This is an author produced version of a paper published in Computer Physics Communications. This paper has been peer-reviewed but does not include the final publisher proof-corrections or journal pagination.

Citation for the published paper:

Jönsson, Per; Gaigalas, Gediminas; Bieron, Jacek; Froese Fischer, Charlotte; Grant, Ian. (2013). New version : grasp2K relativistic atomic structure package. Computer Physics Communications, vol. 84, issue 9, p. null

URL: https://doi.org/10.1016/j.cpc.2013.02.016

Publisher: Elsevier

This document has been downloaded from MUEP (https://muep.mah.se) / DIVA (https://mau.diva-portal.org). 


\title{
New Version: GRASP2K Relativistic Atomic Structure Package
}

\author{
P. Jönsson ${ }^{\text {a,* }}$, G. Gaigalas ${ }^{\text {b }}$, J. Bieron ${ }^{\mathrm{c}}$, C. Froese Fischer ${ }^{\mathrm{d}, \mathrm{e}}$, \\ and I.P. Grant ${ }^{\mathrm{f}}$ \\ ${ }^{a}$ School of Technology, Malmö University, S-20506, Malmö, Sweden \\ ${ }^{\mathrm{b}}$ Vilnius University, Institute of Theoretical Physics and Astronomy, A. Goštauto \\ 12, LT-01108, Vilnius, Lithuania \\ ${ }^{\mathrm{c}}$ Instytut Fizyki imienia Mariana Smoluchowskiego, Uniwersytet Jagiellonski \\ Reymonta 4, 30-059 Kraków, Poland \\ ${ }^{\mathrm{d}}$ Department of Electrical Engineering and Computer Science, Box 1679B, \\ Vanderbilt University, TN 37235, USA \\ e Atomic Physics Division, National Institute of Standards and Technology, \\ Gaithersburg, Maryland 20899-8422, USA \\ ${ }^{\mathrm{f}}$ Mathematical Institute, 24-29 St. Giles, Oxford OX1 3LB, UK
}

\begin{abstract}
A revised version of GrasP2K [P. Jönsson, X. He, C. Froese Fischer, and I.P. Grant, Comput. Phys. Commun. 177 (2007) 597] is presented. It supports earlier non-block and block versions of codes as well as a new block version in which the njgraf library module [A. Bar-Shalom and M. Klapisch, Comput. Phys. Commun. 50 (1988) 375] has been replaced by the librang angular package developed by G. Gaigalas based on the theory of [G.A. Gaigalas, Z.B. Rudzikas, and C. Froese Fischer, J. Phys. B: At. Mol. Phys. 30 (1997) 3747, G. Gaigalas, S. Fritzsche, and I.P. Grant, Comput. Phys. Commun. 139 (2001) 263]. Tests have shown that errors encountered by njgraf do not occur with the new angular package. The three versions are denoted v1, v2, v3, respectively. In addition, in v3, the coefficients of fractional parentage have been extended to $j=9 / 2$, making calculations feasible for the lanthanides and actinides. Changes in v2 include minor improvements. For example, the new version of rci2 may be used to compute QED corrections only from selected orbitals. In v3 a new program, jj2lsj, reports the percentage composition of the wave function in $L S J$ and the program rlevels has been modified to report the CSF with the largest coefficient of an LSJ expansion. The bioscl2 and bioscl3 application programs have been modified to produce a file of transition data with one record for each transition in the same format as in ATSP2K [C. Froese Fischer, G. Tachiev, G. Gaigalas, and M.R. Godefroid, Comput. Phys. Commun. 176 (2007) 559], that identifies each atomic state by the total energy and a label for the CSF with the largest expansion coefficient in $L S J$ intermediate coupling. All versions of
\end{abstract}


the codes have been adapted for 64-bit computer architecture.

Key words: atomic structure calculations, Breit interaction, configuration interaction, correlation, Dirac-Fock theory, energy levels, hyperfine structure, isotope shift, $j j$ coupling, $L S J$ intermediate coupling, multiconfiguration Dirac-Hartree-Fock, nuclear volume effects, QED, relativistic effects in atoms, specific mass shift, transverse photon interactions, transition probabilities, Zeeman effect

PACS: 2.70, 32.10.-f, 31.15Ne, 31.25.-v, 32.30.-r

* Corresponding author: Per.Jonsson@mah.se 


\section{PROGRAM SUMMARY}

Title of the Program: GRASP2K, version 1_1

Catalogue identifier:

ADZL_v1_1

Licensing provisions: no

Programming language: Fortran.

Computer: Intel Xeon, $2.66 \mathrm{GHz}$

Operating system: Suse, Ubuntu, and Debian Linux 64-bit

High-speed storage required: $500 \mathrm{MB}$ or more

No. of bytes in distributed program, including test data, etc.: 51 Mbytes

Distribution format: tar gzip file

Nature of problem:

Prediction of atomic properties - atomic energy levels, oscillator strengths, radiative decay rates, hyperfine structure parameters, Landé $g_{J}$-factors, and specific mass shift parameters - using a multiconfiguration Dirac-Hartree-Fock approach.

Solution method:

The computational method is the same as in the previous GRASP2K [1] version except that for v3 codes the njgraf library module [2] for recoupling has been replaced by librang $[3,4]$.

\section{Restrictions:}

The packing algorithm restricts the maximum number of orbitals to be $\leq 214$. The tables of reduced coefficients of fractional parentage used in this version are limited to subshells with $j \leq 9 / 2$ [5]; occupied subshells with $j>9 / 2$ are, therefore, restricted to a maximum of two electrons. Some other parameters, such as the maximum number of subshells of a configuration state function (CSF) outside a common set of closed shells are determined by a parameter. def file that can be modified prior to compile time.

Unusual features:

The biosc13 program reports transition data in the same format as in ATsP2K [6], and the data processing program tables of the latter package can be used. The tables program takes a name.1sj file, usually a concatenated file of all the .Isj transition files for a given atom or ion, and finds the energy structure of the levels and the multiplet transition arrays. The tables posted at the website 
http://atoms . vuse.vanderbilt. edu are examples of tables produced by the tables program. With the extension of coefficients of fractional parentage to $j=9 / 2$, calculations for the lanthanides and actinides become possible.

Running time:

CPU time required to execute test cases: $70.5 \mathrm{~s}$.

\section{References}

[1] P. Jönsson, X. He, C. Froese Fischer, and I.P. Grant, Comput. Phys. Commun. 177 (2007) 597.

[2] A. Bar-Shalom and M. Klapisch, Comput. Phys. Commun. 50 (1988) 375.

[3] G.A. Gaigalas, Z.B. Rudzikas, and C. Froese Fischer, J. Phys. B: At. Mol. Phys. 30 (1997) 3747.

[4] G. Gaigalas, S. Fritzsche, and I.P. Grant, Comput. Phys. Commun. 139 (2001) 263.

[5] G. Gaigalas, S. Fritzsche, and Z. Rudzikas, At. Data Nucl. Data Tables 76 (2000) 235.

[6] C. Froese Fischer, G. Tachiev, G. Gaigalas, and M.R. Godefroid, Comput. Phys. Commun. 176 (2007) 559.

\section{Introduction}

A revised version of GRASP2K [1] is presented. Some changes are in response to errors that have been reported, whereas others are modifications for improved reliability or extended capability. The previous version maintained the non-block format of GRAsP92 [2] placing such codes in the directory v1. Versions for large-scale computation in block format were retained in the directory v2. In this spirit, we have placed versions using the new library based on the approach of reduced coefficients of fractional parentage $[3,4]$ in directory v3 along with a new jj2ls j program written in Fortran 90 and based on the $L S$ $j j$ transformation matrices for a shell of equivalent electrons [5]. All versions are still included in this new GRASP2K release. 


\section{Installation}

The installation of GRASP2K relies on environment variables. In the new version two environment variables have been added. The first is NEWGRASPLIBS that specifies the libraries to be searched for v3 codes. The second is GRASP_INCLUDES as described below. The environment variables can be set by executing a shell script. Scripts for 64-bit Linux systems using the gfortran, ifort, and pgf95 compiler are available. We recommend the use of the GNU gfortran compiler. Extensive consistency checks of the code have been done with different versions of this compiler, the latest version being gec 4.6.3. The full installation procedure of GRASP2K, including the use of shell scripts to set environment variables, is described in detail in the README file that resides in the main directory, grasp2K_v1_1, of the package.

\section{Parameters in the package}

The GrasP2K routines are designed around a number of parameters as shown in Table 1. These parameter values need to be defined in the parameter.def file prior to compilation. A default copy of the parameter.def file with the values shown in Table 1 is included in this distribution. The makefiles have been modified to search for this file in a directory specified by a new environment variable, GRASP_INCLUDES.

Increasing the values of parameters often extends the range of arrays and hence the memory that is required. For example, the parameter NNNQN defines the maximum number of sub-shells outside the common closed core. The parameter NNNP determines the number of points in a grid. Whereas some parameters are only limited by memory considerations, others like NNNW are limited by the packing algorithm.

\subsection{The GRASP2K radial grid}

The GRASP2K radial grid is determined by the value of RNT, the first non-zero point on the grid, a parameter $\mathrm{H}$, and the number of grid points NNNP. The grid points are then

$$
R(I)=\operatorname{RNT} *(\exp ((I-1) * H)-1), I=1, \ldots, \operatorname{NNNP}
$$

The present defaults are RNT $=2.0 \times 10^{-6}, \mathrm{H}=5.0 \times 10^{-2}$, and NNNP $=590$. 
Table 1

Present parameter values. Except for KEYORB all names of parameters start with NNN.

\begin{tabular}{llll} 
Plant & Meaning or value & $\begin{array}{l}\text { present } \\
\text { value }\end{array}$ & $\begin{array}{l}\text { maximum } \\
\text { value }\end{array}$ \\
\hline \multirow{2}{*}{ KEYORB } & Integral index encoding key & 121 & 215 \\
NNNP & Maximum number of radial tabulation points & 590 & \\
NNN1 & NNNP+10 & 120 & 214 \\
NNNW & Maximum number of relativistic subshells & 119 & 213 \\
NNNWM1 & NNNW-1 & 118 & 212 \\
NNNWM2 & NNNW-2 & 30 & 54 \\
NNNWP & INT (NNNW/4) & 10 & \\
NNNQN & Maximum number of quantum numbers required & & \\
& to completely specify the coupling & 10 & \\
NNNT JV & Maximum number of different $J$ values & & \\
& &
\end{tabular}

The grid defaults may be changed at run time entering $\mathrm{n}$ (no) in response to question about defaults. For $\mathrm{Hg}$, RNT $=1.0 \times 10^{-6}$ and $\mathrm{H}=1.0 \times 10^{-2}$ have been recommended. Many decisions in GRASP2K are based on the parameter $\mathrm{ACCY}=\mathrm{H} * * 6$ (set in the rscf routine getscd.f). For this value of H, ACCY would be $1.0 \times 10^{-12}$. Users should be aware that tests such as for convergence may be as small as $0.001 \times$ ACCY.

\section{The rang angular library}

In v3 programs the rang library for recoupling replaces the njgraf library module [6] used in earlier versions. The rang library is based on the combination of second quantization in the coupled tensorial form, angular momentum theory in three spaces (orbital, spin and quasispin), and a generalized graphical technique [3], and is similar to the ANCO library [7]. In this theory the coefficients of fractional parentage are greatly reduced [4] and have been extended to include $j=9 / 2$. Thus all open $f$-shells can be computed, but correlation subshells with higher $j$ orbital quantum numbers are restricted in occupation to at most two. 
Several codes are available that use the new and extended angular library. When compared with codes based on the njgraf library module there is a considerable speed up. Depending on the case and on the operator the new codes are up to 5 times faster.

\subsection{The jjreduce3 program}

The jjreduce 3 program selects CSFs that have at least one non-zero matrix element with a CSF of a reference list. The program is in non-block form, but has been updated and now uses the rang library to compute the matrix elements.

\subsection{The mcp3 program}

The mcp3 program uses the rang library to generate the angular data needed by rscf2. No code modifications are needed. For consistency, the user may wish to define rscf 3 to be the same as that of rscf2 (copy the executable $\operatorname{rscf} 2$ to $\operatorname{rscf} 3)$.

\subsection{The rci3 program}

The rci3 program is the version of rci2 that uses the new rang library.

Relativistic corrections beyond the Dirac-Coulomb approximation for a many electron system are implemented using assumptions based on one-electron concepts. For example, the self-energy correction is computed from a screenedhydrogenic approximation, a model that does not apply well to correlation orbitals that are far from hydrogenic. To handle this the rci2 and rci3 codes now include an option that limits the self-energy correction to user specified orbitals.

The rci3 includes an option in the non-default mode to shift diagonal energies in the interaction matrix to bring calculated eigenvalue differences in agreement with experimental spectra. This process is referred to as fine-tuning [8].

\subsection{The rhfs3 program}

The rhfs 3 program is the version of rhfs 2 that uses the new rang library. 


\subsection{The biotra3 and bioscl3 programs}

These are versions of the biotra2 and bioscl2 programs that use the rang library.

Additional modifications have been made in biosc13 to produce a name1. name2. (c)t.1sj file in the format of the output of biotr of ATsP2K [9]. This file contains a series of records, one for each transition, that can then be processed by the ATsP2K tables program to produce tables of energy levels and transition tables. These tables can then be used to post results to the MCHF/MCDHF Database [10]. A sample output file from biosc13, taken from the included examples directory, see section 6 , is given in Table 2 . 
Table 2

Sample output file from biosc13. For electric multipole transitions parameters are given in both length form (fourth row of a record) and velocity form (fifth row of a record). dT is the absolute value of the fractional difference of the transition rate AKI in the two forms.

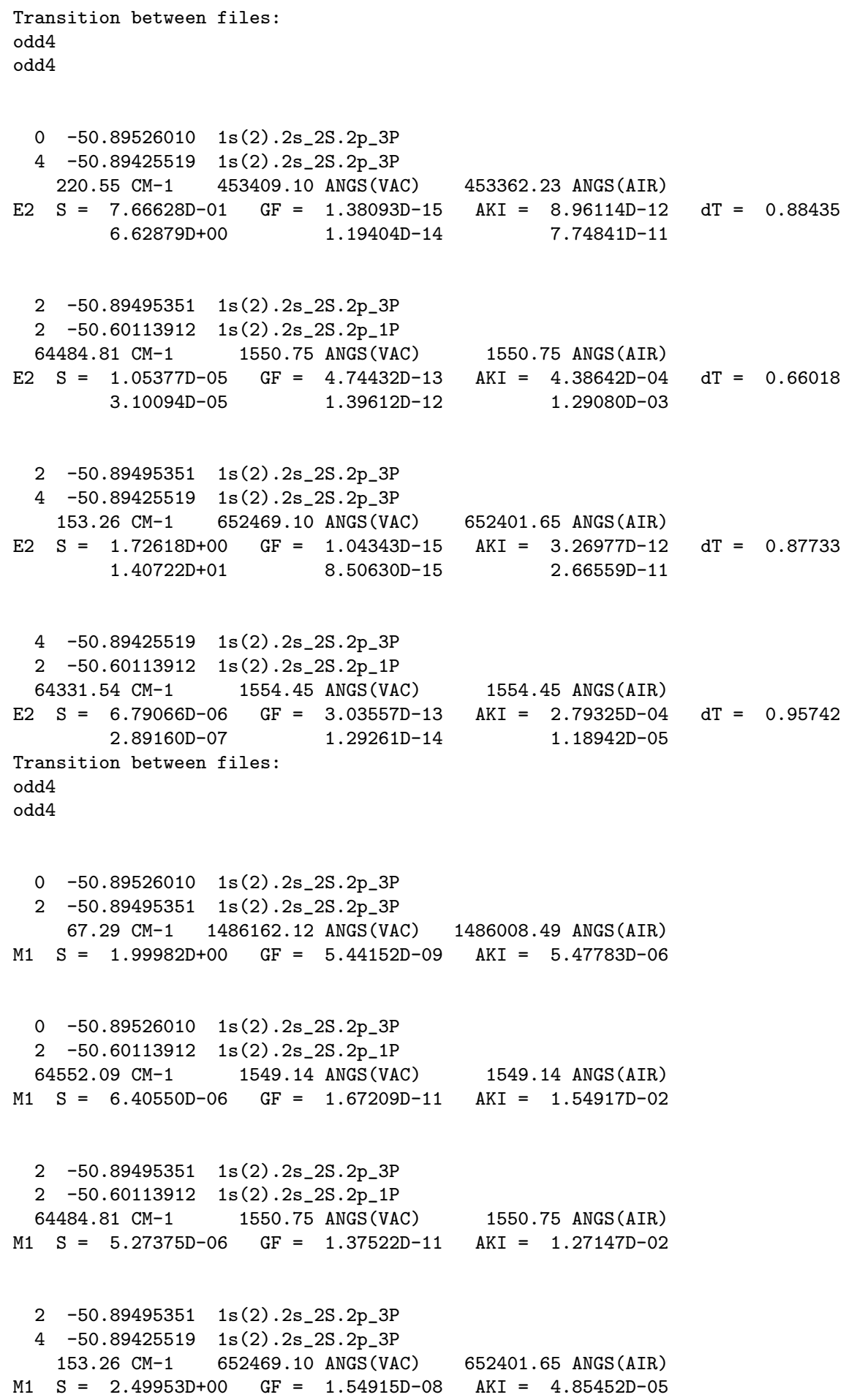




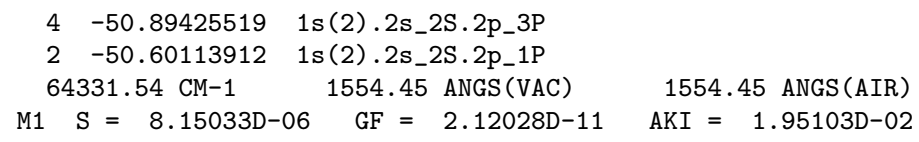

\section{$5 \quad$ New v3 programs}

\subsection{The jj2lsj program}

Unlike the earlier LSJ program [11], that transforms a wave function expanded in a basis of $j j$-coupled configuration state functions (CSFs) to a basis of $L S J$ coupled CSF's, the purpose of the present code is to transform only the most important components of large expansions. In the Atomic Spectra Database (ASD) [12] the composition of a level is frequently given by the two most important components, which in some cases may account for less than $90 \%$ of the wave function. The present code allows the user to select the maximum percentage of the wave function that can be omitted. From the latter information, it is easy to derive the smallest coefficient in the CSF expansion that must be included. However, with many components of about the same size, smaller values may be needed to meet the original objective. In this implementation, the user specifies the CSFs that can be omitted. The remaining CSFs define the basis that is to be transformed. By transforming this basis in decreasing order of importance, the desired percentage of the wave function can be transformed. A second parameter controls the printing of expansion coefficients in the $L S J$ basis and their contribution to the composition of the wave function. The default is to transform at least $99 \%$ of the wave function composition and print components in $L S J$ that contribute more than $0.1 \%$ to the composition. The cut-off for the $j j$-expansion has the value of 0.005 whereas the cut-off for printing is 0.001 .

Table 3 Table showing the transformation of the wave function expansion from a $j j$-coupled basis to an $L S J$-coupled basis for $2 s 2 p{ }^{1,3} P_{1}$ in $N^{+3}$.

jj2lsj: Transformation of ASFs from a jj-coupled CSF basis into an LS-coupled CSF basis (Fortran 95 version) (C) Copyright by G. Gaigalas and Ch. F. Fischer (2011).

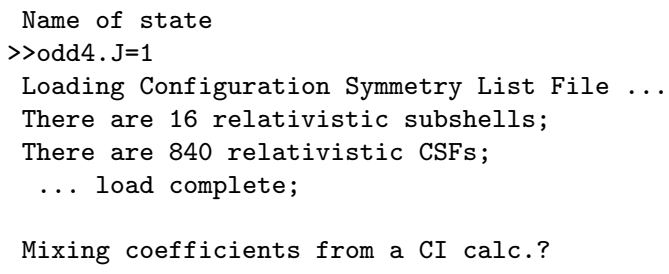




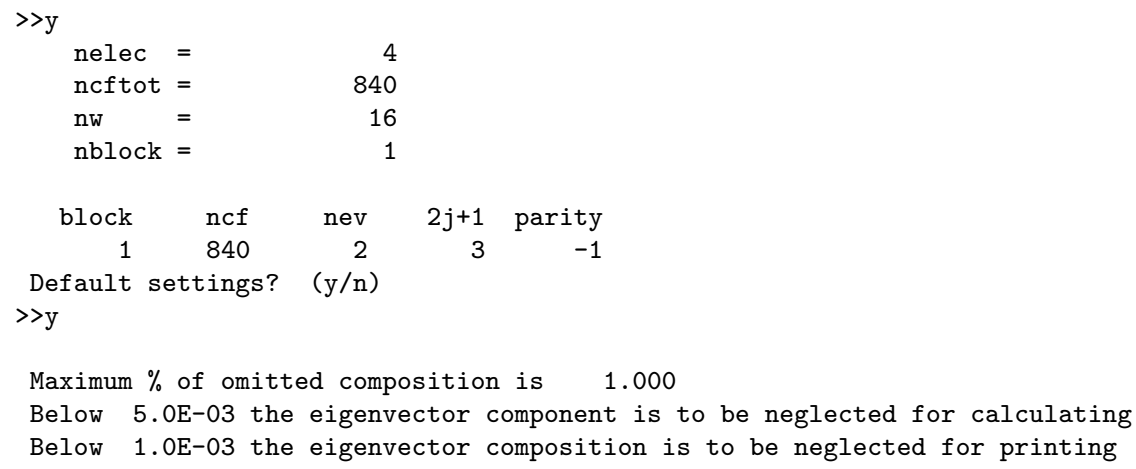

Definition of leading CSF:

$$
\begin{aligned}
& \text { 2) } 1 \mathrm{~s}(2) \quad 2 \mathrm{~s}(1) \quad 2 \mathrm{p}-(1) \\
& \begin{array}{lll}
1 / 2 & 1 / 2 & \\
1 / 2 & & 1
\end{array}
\end{aligned}
$$

Weights of major contributors to ASF in LS-coupling:

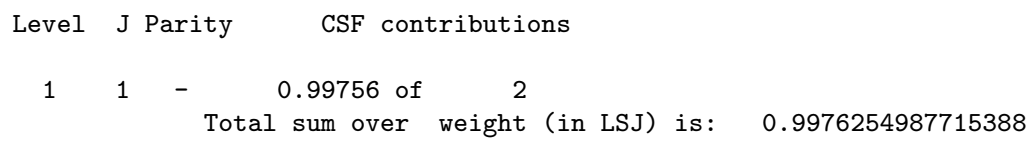

Definition of leading CSF :

Definition of leading CSF:

$$
\begin{aligned}
& \text { 1) } 1 \mathrm{~s}(2) \quad 2 \mathrm{~s}(1) \quad 2 \mathrm{p}(1) \\
& 1 / 2 \quad 3 / 2 \\
& 1 / 2 \quad 1
\end{aligned}
$$

Weights of major contributors to ASF in LS-coupling:
Level J Parity
CSF contributions
0.98444 of $\quad 1 \quad 0.01365$ of 
Definition of leading CSF:

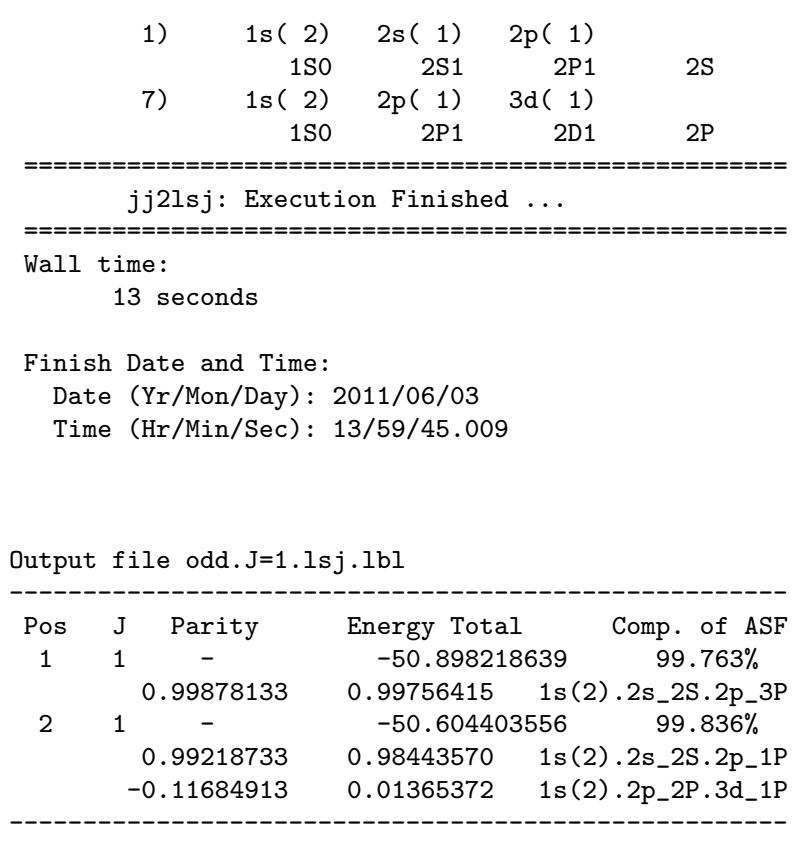

Table 3 shows the execution of the program for the $2 s 2 p{ }^{1,3} P_{1}$ example for $N^{+3}$, built on a basis containing orbitals with principal quantum numbers up to $n=4$, along with the contents of the odd. $\mathrm{J}=1.1 \mathrm{sj} .1 \mathrm{bl}$ output file. For each ASF, the position, $J$, parity, total energy (in hartrees), and percentage of the wave function compositions are provided, followed by a list of coefficients, their squares, and the CSF in $L S J$-coupling.

In the non-default mode, the input queries are:

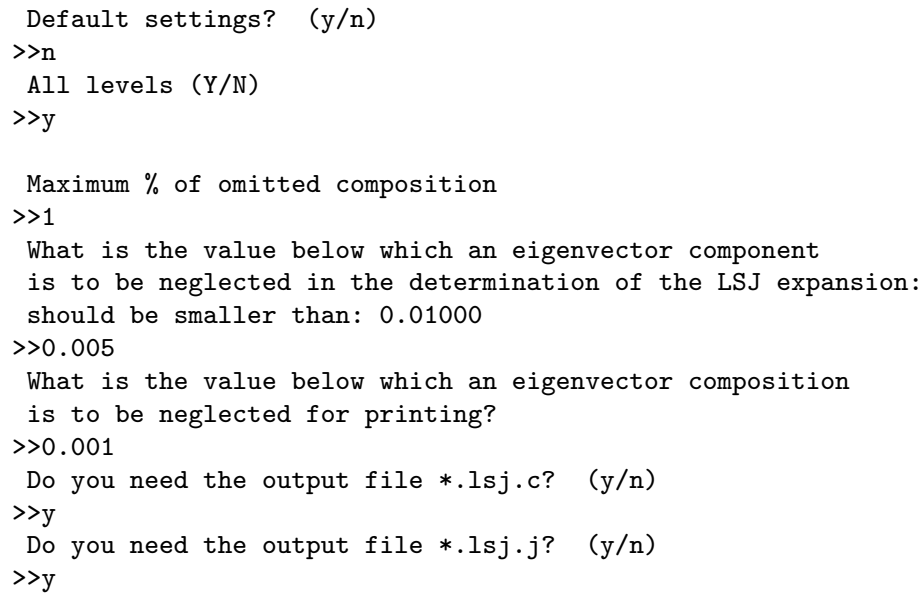

In particular, the user may request a complete transformation, with a list of CSFs in $L S$ in name.lsj.c and their expansion coefficients in name.lsj.j. The two files have the same format as in ATsP2K [9]. Complete expansions 
are feasible only for small expansions.

\section{Modifications to v2 programs}

Several codes contain minor changes.

(1) The rscf2 has had a change that improves convergence. In variational calculations, the diagonal energy parameter may oscillate from one iteration to the next. In getscd.f the damping parameter ODAMP(I) has been changed to an initial value of 0.0 rather than 1.0 and dampck. $f$ modified to change the damping factor only if the oscillations are sufficiently large.

(2) The rci2 program includes the option in the non-default mode to shift diagonal energies in the interaction matrix. As described earlier for rci3 it is now also possible to limit the self-energy correction to user specified orbitals.

(3) The format of rlevels has changed in that the field for the number of levels has been increased to allow for up to 100 levels. In addition, if the name.1sj.lbl files are available for each of the input files, the first label in the name.1sj.Ibl files for the ASF will be appended to the former output.

(4) The biosc12 program has been modified to produce a file in the ATsP $2 \mathrm{~K}$ format as described earlier for biosc13. The output format has been changed: the transition energy is reported in a fixed format to facilitate sorting using the Linux sort command, and the number of digits reported reduced to reflect possible accuracy.

(5) The outputs of the rhfs2 and sms2 programs have been shortened and the programs no longer print information on radial orbitals and energy eigenvalues. The sms2 program is based on the non-relativistic form of the specific mass shift operator. The program is obsolete and is replaced by the Ris program [13], that implements operators to the lowest-order relativistic approximation and to first order in $(m / M)$.

\section{$7 \quad$ Example and manual}

The distributed code includes the previously published example for forbidden E2 and M1 transitions between levels of $2 s 2 p{ }^{1,3} \mathrm{P}$ in $\mathrm{N}^{+3}$. The script files in the examples directory have been modified to use the new codes, when appropriate. The outputs produced from the calculations are found in the output directory. The execution time for the test calculation is $70.5 \mathrm{~s}$. For the new version a comprehensive manual in pdf-format has been prepared with instructions on how to run the codes and interpret the output files. The manual 
is found in the manual directory and contains examples from calculations for $1 s^{2} 2 s^{2} S, 1 s^{2} 2 p^{2} P$ in Li I, $2 s 2 p{ }^{1,3} P$ in B II, and $2 s^{2} 2 p^{3}$ and $2 p^{5}$ in Si VIII. In addition there is a larger case study of the $2 s^{2} 2 p$ and $2 s 2 p^{2}$ states in Mo XXXVIII. The examples in the manual can also be run using script files. These reside in the example1, example2, example3, and casestudy directories. The outputs produced from the calculations described in the manual are found in the corresponding output directories.

\section{Future releases}

To make the GrASP2K package easier to maintain and update, future releases will be based on Fortran 2003 and a more modular structure such as the one found in RATIP [14]. Work along these lines have already begun.

\section{Sample Disclaimer}

Certain commercial equipment, instruments, software, or materials are identified in this paper in order to specify the computational procedure adequately. Such identification is not intended to imply recommendation or endorsement by the National Institute of Standards and Technology, nor is it intended to imply that the materials or equipment identified are necessarily the best available for the purpose.

\section{Acknowledgments}

PJ, GG, and JB acknowledge support from the Visby program of the Swedish Institute. JB acknowledges support by the Polish Ministry of Science and Higher Education (MNiSW) in the framework of the project No. N N202 014140 awarded for the years 2011-2014 as well as by the European Regional Development Fund in the framework of the Polish Innovation Economy Operational Program (contract no. POIG.02.01.00-12-023/08).

\section{References}

[1] P. Jönsson, X. He, C. Froese Fischer, and I.P. Grant, Comput. Phys. Commun. 177 (2007) 597. 
[2] F.A. Parpia, C. Froese Fischer, and I.P. Grant, Comput. Phys. Commun. 94 (1996) 249.

[3] G.A. Gaigalas, Z.B. Rudzikas, and C. Froese Fischer, J. Phys. B: At. Mol. Phys. 30 (1997) 3747.

[4] G. Gaigalas, S. Fritzsche, and Z. Rudzikas, At. Data Nucl. Data Tables 76 (2000) 235.

[5] G. Gaigalas, T. Zalandauskas, and Z. Rudzikas, At. Data Nucl. Data Tables 84 (2003) 99.

[6] A. Bar-Shalom and M. Klapisch, Comput. Phys. Commun. 50 (1988) 375.

[7] G. Gaigalas, S. Fritzsche, and I.P. Grant, Comput. Phys. Commun. 139 (2001) 263.

[8] A. Hibbert, Phys. Scr. T 65 (1996) 104.

[9] C. Froese Fischer, G. Tachiev, G. Gaigalas, and M R. Godefroid, Comput. Phys. Commun. 176 (2007) 559.

[10] C. Froese Fischer, and G. Tachiev, MCHF/MCDHF Collection (http://physics.nist.gov/mchf)

[11] G. Gaigalas, T. Zalandauskas, and S. Fritzsche, Comput. Phys. Commun. 157 (2004) 239.

[12] Yu. Ralchenko et al., NIST Atomic Spectra Database (http://physics.nist.gov/asd)

[13] C. Nazé et al. Comput. Phys. Commun. submitted 2012.

[14] S. Fritzsche, Comput. Phys. Commun. 183 (2012) 1525. 